\title{
88. DSK: Datenschutz erfordert unabhängige Kontrollbehörden
}

Personenbezogene Daten treten immer stärker in den Fokus von öffentlichen und privaten Stellen, die sie in großem Stil erfassen, speichern und verarbeiten. Dabei wird häufig die Sammelwut nur durch die technischen Möglichkeiten begrenzt; ein Ende ist nicht abzusehen. Immer schwerer wird es, der digitalen Technik und der Kapitalisierung von Daten die begrenzende Funktion der Rechte Betroffener entgegenzustellen. Folgerichtig zielen die Forderungen der 88. Konferenz der Datenschutzbeauftragten des Bundes und der Länder zum größten Teil auf bessere Kontrollinstrumente und -möglichkeiten durch unabhängige Datenschutzbehörden ab.

Die 88. Konferenz der Datenschutzbeauftragten des Bundes und der Länder hat im Einzelnen folgende Entschließungen ${ }^{1}$ gefasst: Effektive Kontrolle der Nachrichtendienste herstellen!

Die Datenschutzkonferenz fordert die Gesetzgeber dazu auf, die Datenschutzbeauftragten des Bundes und der Länder sachgerecht auszustatten. Nur so können sie ihrer Kontrollfunktion, die nicht zuletzt vom Bundesverfassungsgericht im Urteil zum Antiterrordateigesetz unterstrichen wurde, nachkommen. Gleichzeitig gilt es, künftig die Datenschutzbeauftragten bei der Überwachung von Nachrichtendiensten zu beteiligen und ihre Expertise zu nutzen.

Marktmacht und informationelle Selbstbestimmung

Die deutsche Monopolkommission hat für eine verstärkte Kooperation von Datenschutz- und Wettbewerbsbehörden plädiert. Die Datenschutzkonferenz schließt sich dem an. Zunehmende Unternehmenskäufe vor allem in der Internetwirtschaft, die wachsende Bedeutung von Big Data sowie die Verflechtung von Internet und Smart Home fordern gemeinsame Strategien.

Unabhängige und effektive Datenschutzaufsicht ist für Grundrechtschutz unabdingbar

Die Konferenz der Datenschutzbeauftragten des Bundes und der Länder begrüßt den Gesetzesentwurf der Bundesregierung, der auf eine Stärkung der Unabhängigkeit der Bundesbeauftragten abzielt. Eine unabhängige Datenschutzaufsicht kann jedoch nur erfolgen, wenn der Behörde ausreichende Personal- und Sachmittel zur Verfügung gestellt werden. Insbesondere muss sich der stetige Aufgabenzuwachs auch in der personellen Ausstattung widerspiegeln. Das gilt auch gerade für viele Datenschutzbehörden in den Ländern, die oft defizitär ausgestattet sind und damit die Grundrechte Betroffener nur unzureichend schützen können.

Recht auf Sperrung von Suchergebnissen bei Anbietern von Suchmaschinen

Die Datenschutzkonferenz begrüßt die Entscheidung des Europäischen Gerichtshofs zur Löschung von Treffern in der Suchmaschine von Google als „fundamentalen Beitrag zum Schutz der Persönlichkeitsrechte im Internet“. Das in dem Urteil geprägte „Recht, schwer gefunden zu werden“ muss nun - der globalen Natur des Internet entsprechend - weltweit umgesetzt werden.

Datenschutz im Kraftfahrzeug

Moderne Kraftfahrzeuge sind längst auch zu umfassenden Datenspeichern geworden. Fahrverhalten, Aufenthaltsorte und Bewegungen des Fahrers oder der Fahrerin können problemlos ausgelesen, zu gänzlich unterschiedlichen Zwecken verwendet und zu Persönlichkeitsprofilen verknüpft werden. Die Datenschutzkonferenz fordert die Automobilindustrie sowie Händler und Werkstätten dazu auf, das Recht auf informationelle Selbstbestimmung auch im Kraftfahrzeug zu gewährleisten.

Dazu Johannes Caspar, der amtierende Vorsitzende der Konferenz: „Datenschutz ist Grundrechtsschutz. Dieser ist in der digitalen Welt immer schwerer zu gewährleisten. Datenschutzbehörden stehen öffentlichen und privaten Stellen gegenüber, die oft auf nahezu unerschöpfliche personelle und finanzielle Ressourcen zurückgreifen können. Ein wirksamer Grundrechtsschutz des Einzelnen gegenüber den immer tiefer in das Privatleben eingreifenden Technologien und einer Algorithmisierung unserer Lebenswelt bei der Nutzung digitaler Technologien erfordert gerade auch die Stärkung einer unabhängigen Datenschutzaufsicht in Bund und Ländern sowie eine den wachsenden Herausforderungen angemessene Ausstattung."

1 Siehe Dokumentation in diesem Heft, S. $42-44$ 